\title{
Modified FDTD Near-to-Far-Field Transformation for Improved Backscattering Calculation of Strongly Forward-Scattering Objects
}

\author{
$\mathrm{Xu}$ Li, Member, IEEE, Allen Taflove, Fellow, IEEE, and Vadim Backman
}

\begin{abstract}
We propose a simple yet effective modification to significantly improve the accuracy of the finite-difference time-domain (FDTD) near-to-far-field (NTFF) transformation for calculating the backscattering of objects having strong forward-scattering lobes. In the modified approach, we merely omit integrating over the near-field plane located in the forward-scattering region. We validate the proposed approach via comparison with rigorous analytical solutions of plane waves scattered by single and multiple dielectric spheres.
\end{abstract}

Index Terms-Finite-difference time-domain (FDTD) methods, near-to-far-field (NTFF) transformation, scattering.

\section{INTRODUCTION}

$\mathbf{S}$ INCE introduced in the early 1980s [1], the near-to-far-field (NTFF) transformation has been routinely used in the finite-difference time-domain (FDTD) method [2] for calculating antenna scattering patterns and radar cross sections. The standard NTFF formulation is based on the surface equivalence theorem [2]. In this approach, the scattered $\mathrm{E}$ and $\mathrm{H}$ fields tangential to a closed virtual surface surrounding the structure of interest are computed via FDTD and integrated over the entire surface to provide the far-field response. Since the virtual surface is independent of the geometry of the enclosed scatterer, it is usually chosen as a rectangular shape to conform to the standard Cartesian FDTD grid. Using the standard FDTD-NTFF scheme, far-field scattering amplitudes and phases can be calculated for any scattering angles. In this paper, we focus our discussion on the calculation of far-field backscattering spectra, which are important for many applications.

Experimentally, backscattered signals are obtained with a monostatic radar configuration. These signals can be used to detect and identify scattering structures in radar-imaging and remote-sensing applications [3], [4]. More recently, it has been demonstrated that backscattered light spectra, which can be measured noninvasively from biological tissues and cells, can provide valuable medical diagnostic information

Manuscript received September 17, 2004; revised December 16, 2004. This work was supported by the National Science Foundation under Grants BES0238903 and ACI-0219925.

X. Li and V. Backman are with the Department of Biomedical Engineering, Northwestern University, Evanston, IL 60208 USA (e-mail: xuli@northwestern.edu; v-backman@northwestern.edu).

A. Taflove is with the Department of Electrical and Computer Engineering, Northwestern University, Evanston, IL 60208 USA (e-mail: taflove@ece.northwestern.edu)

Digital Object Identifier 10.1109/LAWP.2005.845038
[5], [6]. In fact, applications in optical tissue diagnosis have motivated a series of recent FDTD-based numerical studies for understanding light scattering by complex material shapes in the 1-10 wavelength $(\lambda)$ size [7]-[9]. Such objects are generally characterized by strong forward-scattering lobes [4]. However, we have observed from our FDTD numerical studies that accurate calculation of backscattering spectra is challenging for such objects. Here, converged backscattering results require a spatial resolution that is much finer than $\lambda / 20$, which usually suffices for the forward-scattering calculation. This requirement for very fine resolution poses a significant computational burden.

In this paper, we present a numerical investigation which leads to a simple technique to improve the accuracy of the far-field backscattering calculation in the FDTD-NTFF method for strongly forward-scattering objects. We first show that, for an object with strong forward scattering, a small percentage numerical error in calculating the fields along the NTFF plane located in the forward-scattering region results in a large discrepancy in the calculated far-field backscattering. Based on these numerical observations, we propose a simple yet effective modification to significantly improve the accuracy of the FDTD-NTFF transformation for calculating the backscattering of such objects. In our modified approach, we merely omit integrating over the NTFF plane located in the forward-scattering region. We validate the proposed approach via comparison with rigorous analytical solutions of plane waves scattered by single and multiple dielectric spheres.

\section{ACCuRACy Degradation IN the FDTD BaCkSCATtERING CALCULATION FOR STRONGLY FORWARD-SCATTERING OBJECTS}

We first illustrate the accuracy degradation in the FDTD farfield backscattering calculation for strongly forward-scattering objects via comparison with the exact solution (Mie theory) [4] for dielectric spheres. In our FDTD simulations, the geometry of a dielectric sphere is imported to a cubic-cell three-dimensional FDTD spatial lattice ( $\Delta x=\Delta y=\Delta z=25 \mathrm{~nm}$ ) using a staircasing scheme. We terminate the FDTD lattice using a Berenger perfectly matched layer (PML) absorbing boundary condition [10]. Using the pure-scattered field formulation [11], we excite the sphere with an impulsive plane wave having spectral content in the 500-1000 nm wavelength range. The phasor-domain scattered-field response is extracted via a discrete Fourier transform of the time-domain data recorded on all six NTFF planes, normalized by the source spectrum. Then, we integrate these 


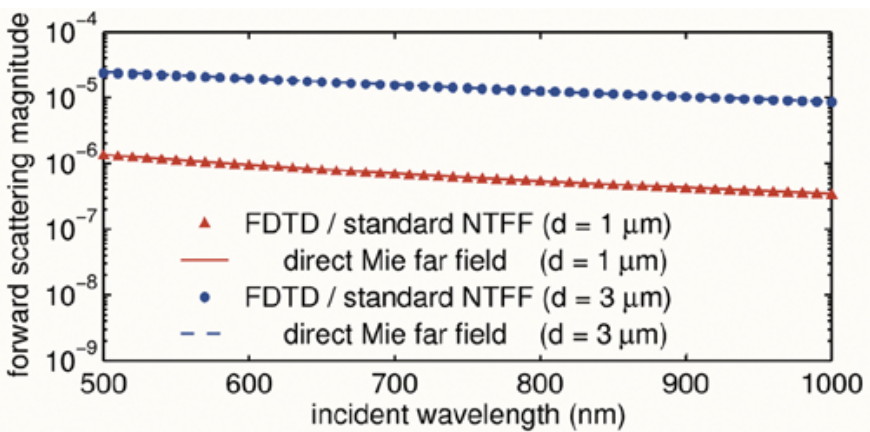

(a)

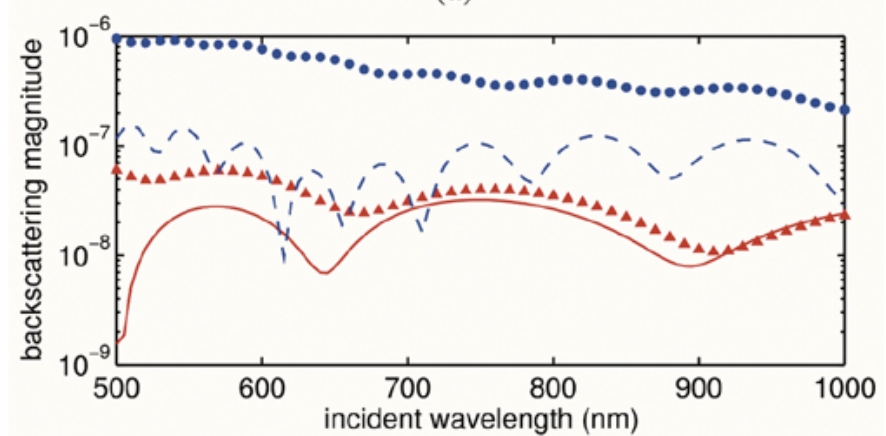

(b)

Fig. 1. Far-field scattering spectra of $1-\mu \mathrm{m}$ and $3-\mu \mathrm{m}$ diameter dielectric spheres with $\epsilon_{r}=1.21$. (a) Demonstration of the accuracy of the FDTD-calculated forward scattering when applying the standard NTFF transformation. (b) Demonstration of the inaccuracy of the FDTD-calculated backscattering when applying the standard NTFF transformation.

phasor-domain field components to obtain the far-field scattered amplitudes in both the forward and backward directions.

Fig. 1 compares the FDTD-NTFF calculated spectra with Mie theory for two $\epsilon_{r}=1.21$ dielectric spheres of diameters $d=1 \mu \mathrm{m}$ and $d=3 \mu \mathrm{m}$. As illustrated in Fig. 1(a), the FDTD-calculated forward-scattering spectra closely match the analytical values with a 5\% maximum error for both sphere diameters. However, as shown in Fig. 1(b), the FDTD-calculated backscattering spectra deviate significantly from the analytical values. The accuracy degradation in the backscattering calculation is more evident for the larger sphere, which has a stronger forward-scattering lobe. We note that our grid resolution, on the order of $\lambda / 20$ ( $25 \mathrm{~nm}$ in this case), is commonly used in FDTD simulations.

\section{THE SOURCES OF ERRORS OF THE STANDARD FDTD-NTFF BACKSCATTERING CALCULATION}

We next investigate possible causes of the poor quality of the FDTD far-field backscattering calculation. In the standard FDTD-NTFF method, errors in the far-field calculation can be traced to two sources: the numerical error in calculating the near-field components used for the NTFF virtual-surface integration, and the error caused by the discrete numerical integration over the virtual surface. To separate these two effects, we again focus our discussion on the problem of plane wave scattering by spheres, where analytical solutions are available to serve as benchmark data.

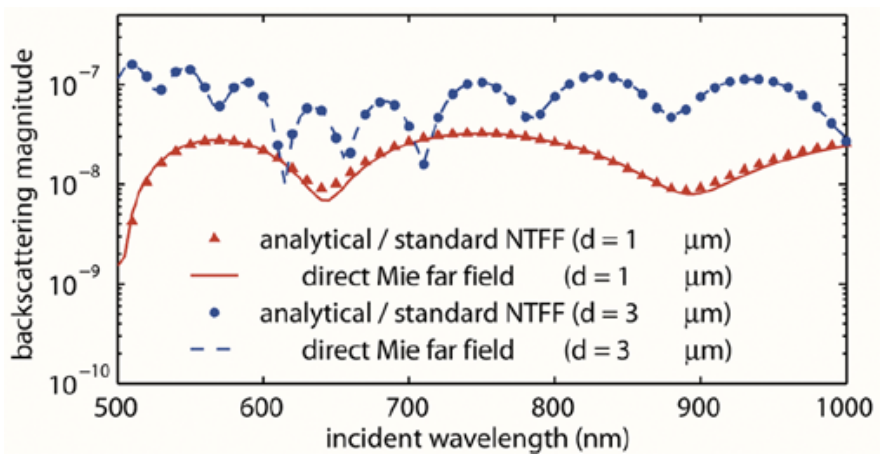

Fig. 2. Demonstration of the validity of the standard NTFF integration procedure when using exact (analytical) near-field data. Far-field backscattering spectra are shown for $1-\mu \mathrm{m}$ and $3-\mu \mathrm{m}$ diameter dielectric spheres with $\epsilon_{r}=1.21$

The Lorentz-Mie theory is well known for providing rigorous solutions for far-field scattering properties of spheres [4]. Less well known is that this theory also provides rigorous near-field solutions. By computing the exact near-field components along the NTFF virtual surface and then numerically integrating them to generate the far-field data, we are able to isolate the effect of the discrete numerical integration over the virtual surface [12].

Fig. 2 shows the results of far-field backscattering calculations implemented by integrating the exact near-field components for the dielectric spheres examined in the two cases of Fig. 1. The exact near-field components are calculated at the same discrete spatial positions as for the FDTD simulations. Then, the discrete NTFF numerical integration is performed following the same procedure as for the results shown in Fig. 1(b). We see that there is much better agreement with the direct exact Mie far-field calculations for both sphere diameters. Note that the only change here relative to Fig. 1(b) is that the FDTD-calculated near-field components are replaced with exact analytical values. Based on these results, it is evident that the poor calculation accuracy exhibited in Fig. 1(b) is primarily caused by the inaccuracy of the FDTD near-field calculation itself.

We next examine the FDTD-calculated field distribution and its associated errors via comparison with the exact solution at two key planes of the rectangular NTFF virtual surface, as shown in Fig. 3(a): the forward NTFF plane and the backward NTFF plane. Fig. 3(b) and (c) shows E-field distributions at the forward and backward NTFF planes bracketing a 3- $\mu \mathrm{m}$ dielectric sphere for a $\lambda=750 \mathrm{~nm}$ illumination. With a diameter $d=4 \lambda$ and a permittivity $\epsilon_{r}=1.21$, this sphere has a strong forward-scattering lobe. Therefore, the field magnitude at the forward NTFF plane is much stronger than at the backward NTFF plane, as illustrated by the color scales of Fig. 3(b) and (c). Fig. 3(d) and (e) shows the magnitude of the error of the FDTD-computed E-field compared with the exact solution. For both the forward and backward planes, the average relative errors are less than $5 \%\left(\left.\left(\overline{\Delta_{E}} / \bar{E}\right)\right|_{\text {fwd }}=2.4 \%\right.$ and $\left.\left.\left(\overline{\Delta_{E}} / \bar{E}\right)\right|_{\mathrm{bck}}=4.2 \%\right)$. However, the absolute error of the E-field calculation along the forward plane is much greater than the absolute error on the backward plane due to the strong forward-scattering lobe. In fact, for the example shown in Fig. 3, the error magnitudes along the forward plane $\left(\left(\left.\overline{\Delta_{E}}\right|_{\text {fwd }}=0.023 \mathrm{~V} / \mathrm{m}\right)\right.$ are comparable in magnitude to the 


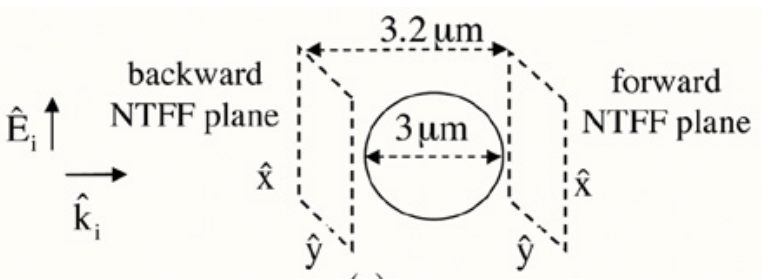

(a)

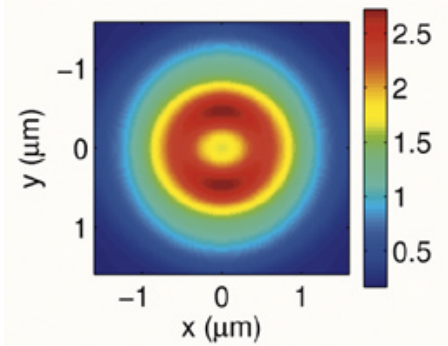

(b)

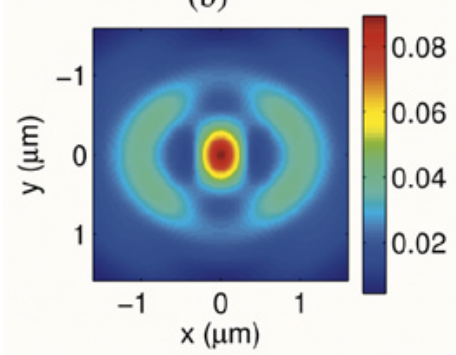

(d)

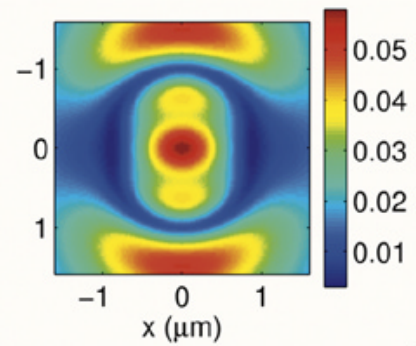

(c)

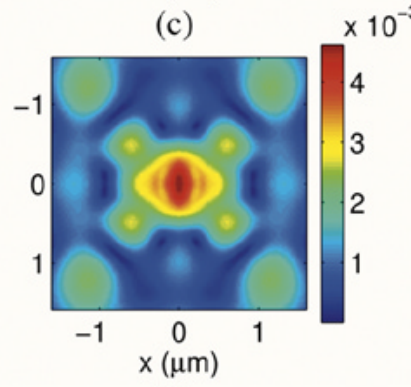

(e)
Fig. 3. Distribution of scattered E-field magnitude on NTFF surface for a 3- $\mu \mathrm{m}$ diameter dielectric sphere with $\epsilon_{r}=1.21$ for $\lambda=750 \mathrm{~nm}$ incident wavelength. (a) Problem geometry. (b) Analytical E-field distribution on the forward NTFF plane. (c) Analytical E-field distribution on the backward NTFF plane. (d) Absolute error of the FDTD-calculated E-field magnitude distribution on the forward NTFF plane. (e) Absolute error of the FDTD-calculated E-field magnitude distribution on the backward NTFF plane.

total value of the scattered fields along the backward plane $\left(\left.\bar{E}\right|_{\mathrm{bck}}=0.025 \mathrm{~V} / \mathrm{m}\right)$. Therefore, it is logical to postulate that the numerical error in the field calculation in the FDTD calculation along the forward plane can significantly contaminate the near-to-far-field transformation in the backscattering direction.

Based on additional numerical experiments, we determine that further optimization of the PML layer or using permittivity averaging to smooth the staircasing does not provide substantial improvement in our case. In order to improve the quality of the near-field calculations, we need to either increase the grid resolution or implement an alternative FDTD scheme with a higher order of accuracy. These approaches inevitably require more computational resources and/or a higher level of coding complexity.

\section{A Simple TeChNique For ACCURACy IMPROVEMENT IN FAR-FIELD BACKSCATTERING CALCULATION}

Instead of trying to improve the near-field calculation, we propose a simple technique to circumvent the problem of significant numerical error in the FDTD field calculations along the forward plane of the NTFF virtual surface. In order to avoid the error contamination in the backscattering calculation, we simply omit the forward plane of the virtual surface from the NTFF surface integration when calculating the far-field backscattering.

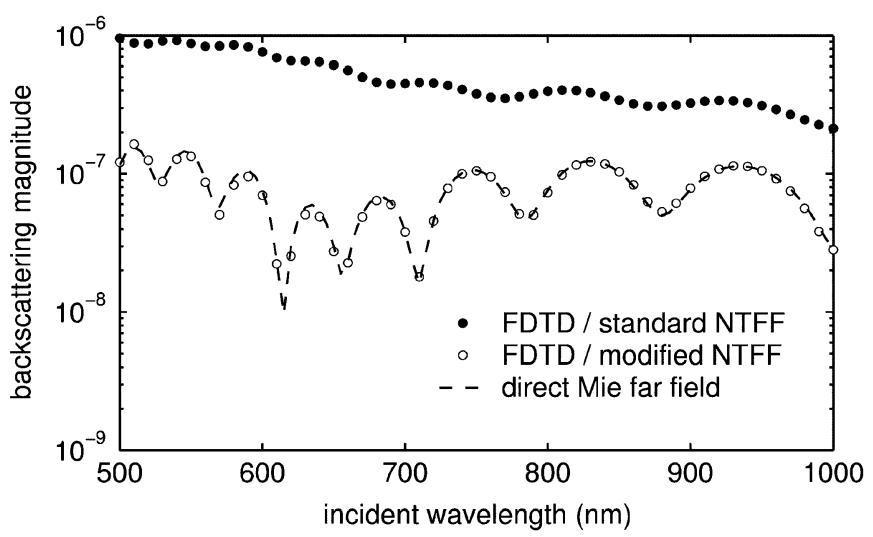

(a)

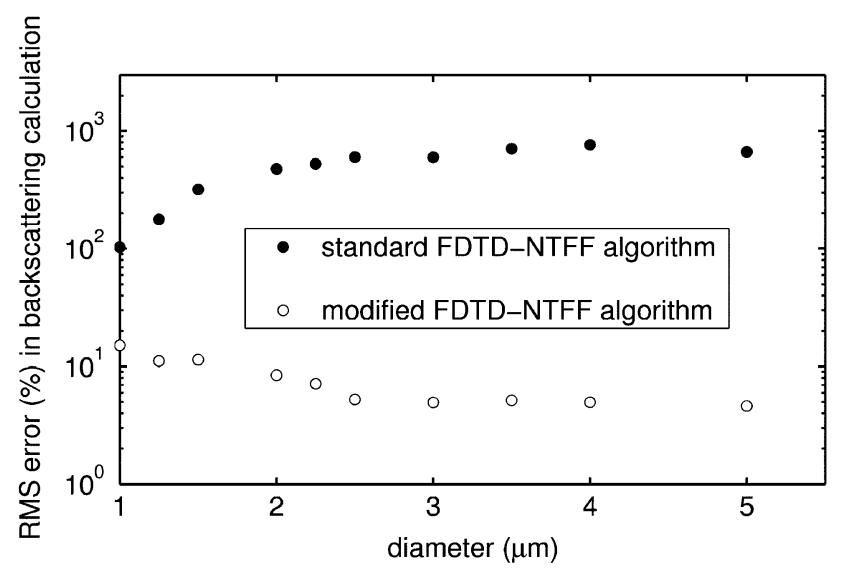

(b)

Fig. 4. Improved accuracy of the modified NTFF algorithm for FDTD calculation of the backscattering spectra of dielectric spheres. (a) Backscattering spectrum over a 500-1000 nm incident wavelength range for a 3- $\mu \mathrm{m}$ diameter dielectric sphere with $\epsilon_{r}=1.21$. (b) RMS error of backscattering calculations over the $500-1000 \mathrm{~nm}$ incident wavelength range as functions of sphere diameter for dielectric spheres.

This approach is obviously an approximation to the rigorous surface equivalence theorem, wherein integration over a closed virtual surface completely surrounding the scattering object is required. However, our approximation can be justified by the fact that the Poynting vectors of scattered waves propagating through the forward plane of the NTFF virtual surface approximately point in the forward direction, and, therefore, should have only a small contribution to the far-field backscattering. Our hypothesis is that, for a strongly forward-scattering object, the improved accuracy in omitting the forward-plane field integration may substantially outweigh the loss of accuracy caused by the incomplete surface integration.

We now present numerical evidence that support our hypothesis. Fig. 4(a) compares the backscattering spectra calculated by the original FDTD-NTFF method and our modified approach for the $d=3 \mu \mathrm{m}$ sphere of Fig. 1. We see that, by omitting the forward plane of the virtual surface from the NTFF surface integration, we considerably improve the accuracy of the backscattering spectrum calculation at all wavelengths examined with no additional requirement for computational resources. Fig. 4(b) repeats the calculation for a series of dielectric spheres of diameters ranging from $1 \mu \mathrm{m}$ to $5 \mu \mathrm{m}$. Here, we used a root-mean-square (rms) error functional to characterize 


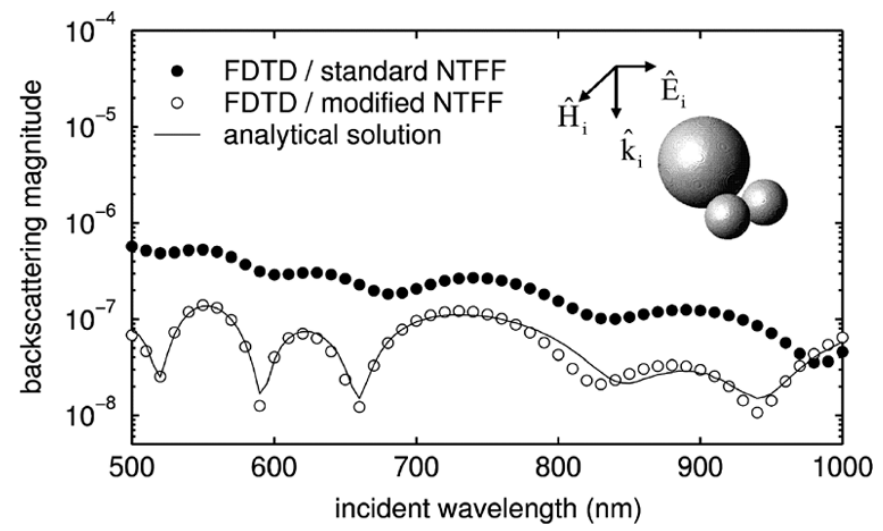

Fig. 5. Improved accuracy of the modified NTFF algorithm for FDTD calculation of the backscattering spectrum of a triplet of dielectric spheres with $\epsilon_{r}=1.21, d_{1}=2 \mu \mathrm{m}$, and $d_{2}=d_{3}=1 \mu \mathrm{m}$.

the accuracy of the FDTD-calculated backscattering spectrum comparing with the far-field analytical solutions. In general, the forward-scattering lobe gets stronger as the size of the dielectric sphere increases. Consequently, the accuracy of the original FDTD-NTFF backscattering calculation deteriorates with increasing size of the sphere. However, omitting the integration over the forward NTFF plane gives substantially improved accuracy, especially for larger spheres, where the effect of staircasing is less prominent. The RMS error of the calculated backscattering spectrum stabilizes at about $2 \%$ for larger spheres for the modified FDTD-NTFF method. This is about $1 / 100$ th of the RMS error of the original FDTD-NTFF approach.

As an additional validation, we calculate the backscattering spectra of aggregations of dielectric spheres using both the original and the modified FDTD-NTFF methods, and compare to the analytical values. A rigorous analytical solution based on an extension of Mie theory for plane-wave scattering by an aggregation of spheres has been developed, implemented, and validated by $\mathrm{Xu}$ et al. [13]. This solution provides us with benchmark data for evaluating the calculation of scattering by nonsymmetrical structures. We have compared the results of our modified FDTD-NTFF technique with the analytical solution of Xu et al. for several different aggregations of dielectric spheres with total dimensions spanning up to $10 \lambda$. An RMS accuracy for the backscattering spectrum of better than $10 \%$ has been observed for all cases examined. Fig. 5 illustrates an example where we calculate the backscattering spectra of a three-sphere aggregation. Similar to our findings for the single dielectric sphere, it is clear that omitting the forward plane from the NTFF integration greatly improves the accuracy in calculating backscattering.

\section{SUMmARY AND DISCUSSION}

In this paper, we analyzed the accuracy degradation in the FDTD-NTFF far-field backscattering calculation for strongly forward-scattering objects. We found that numerical errors in the FDTD field calculation along the forward plane of the NTFF virtual surface contaminate the backscattering calculation. We proposed to improve the accuracy of the backscattering calculation by omitting the forward plane of the NTFF virtual surface from the NTFF surface integration. For both isolated dielectric spheres and aggregations of dielectric spheres, we demonstrated that our proposed approach significantly improves the accuracy of the backscattering calculation with zero increase of the computational burden.

In addition to having applications in numerical studies related to biophotonics, the method proposed in this paper may provide improved FDTD modeling of the monostatic radar cross section of certain types of low-observable aerospace vehicles.

\section{ACKNOWLEDGMENT}

The authors thank Dr. S. Zou and Z. Chen for providing portions of the near-field Mie code. They would also like to thank Dr. Y. L. Xu for making publicly available his Fortran codes for multiparticle light scattering calculations.

\section{REFERENCES}

[1] K. R. Umashankar and A. Taflove, "A novel method to analyze electromagnetic scattering of complex objects," IEEE Trans. Electromagn. Compat., vol. 24, no. 4, pp. 397-405, Nov. 1982.

[2] A. Taflove and S. Hagness, Computational Electrodynamics: The FiniteDifference Time-Domain Method, 2nd ed. Boston, MA: Artech House, 2000.

[3] L. Tsang, J. A. Kong, and R. T. Shin, Theory of Microwave Remote Sensing. New York: Wiley, 1985.

[4] A. Ishimaru, Wave Propagation and Scattering in Random Media, 2nd ed. New York: Academic, 1978.

[5] Y. L. Kim, Y. Liu, R. K. Wali, H. K. Roy, M. J. Goldberg, A. K. Kromine, K. Chen, and V. Backman, "Simultaneous measurement of angular and spectral properties of light scattering for characterization of tissue microarchitecture and its alteration in early precancer," IEEE J. Sel. Topics Quantum Electron., vol. 9, no. 2, pp. 243-256, Mar. 2003.

[6] H. K. Roy, Y. Liu, R. Wali, Y. L. Kim, A. K. Kromine, M. J. Goldberg, and V. Backman, "Four-dimensional elastic light-scattering fingerprints as preneoplastic markers in the rat model of colon carcinogenesis," Gastroenterology, vol. 126, no. 4, pp. 1071-1081, Apr. 2004.

[7] R. Drezek, A. Dunn, and R. Richards-Kortum, "Light scattering from cells: Finite-difference time-domain simulations and goniometric measurements," Appl. Opt., vol. 38, no. 16, pp. 3651-3661, Jun. 1999.

[8] X. Li, Z. Chen, A. Taflove, and V. Backman, "Analytical techniques to address the forward and inverse problems in light scattering by irregularly shaped particles," Opt. Lett., vol. 29, pp. 1239-1241, 2004.

[9] — "Equiphase-sphere approximation for light scattering by stochastically inhomogeneous microparticles," Phys. Rev. E, vol. 70, no. 5, p. $056610,2004$.

[10] J. P. Berenger, "A perfectly matched layer for the absorption of electromagnetic waves," J. Comput. Phys., vol. 114, no. 2, pp. 185-200, Oct. 1994.

[11] R. Holland, L. Simpson, and K. S. Kunz, "Finite-difference analysis of EMP coupling to lossy dielectric structures," IEEE Trans. Electromagn. Compat., vol. 22, no. 3, pp. 203-209, Aug. 1980.

[12] P. W. Zhai, Y. K. Lee, G. W. Kattawar, and P. Yang, "Implementing the near to far-field transformation in the finite-difference time-domain method," Appl. Opt., vol. 43, no. 18, pp. 3738-3746, Jun. 2004.

[13] Y. L. Xu and R. T. Wang, "Electromagnetic scattering by an aggregate of spheres: Theoretical and experimental study of the amplitude scattering matrix," Phys. Rev. E, vol. 58, no. 3, pp. 3931-3948, 1998. 